\title{
MFV reductions of MSSM parameter space
}

\author{
S.S. AbdusSalam, ${ }^{a, b}$ C.P. Burgess ${ }^{c, d, e}$ and F. Quevedo ${ }^{b, f}$ \\ ${ }^{a}$ INFN - Sezione di Roma, \\ P.le A. Moro 2, I-00185 Roma, Italia \\ ${ }^{b}$ The Abdus Salam ICTP, \\ Trieste, Italy \\ ${ }^{c}$ Department of Physics \& Astronomy, McMaster University, \\ Hamilton ON, Canada \\ ${ }^{d}$ Perimeter Institute for Theoretical Physics, \\ Waterloo, ON, Canada \\ e Division PH-TH, CERN, \\ CH-1211, Genève 23, Suisse \\ ${ }^{f}$ DAMTP, Cambridge University, \\ Cambridge, U.K. \\ E-mail: Shehu.AbdusSalam@Roma1.infn.it, \\ cburgess@perimeterinstitute.ca, F.Quevedo@damtp.cam.ac.uk
}

ABSTRACT: The 100+ free parameters of the minimal supersymmetric standard model (MSSM) make it computationally difficult to compare systematically with data, motivating the study of specific parameter reductions such as the cMSSM and pMSSM. Here we instead study the reductions of parameter space implied by using minimal flavour violation (MFV) to organise the R-parity conserving MSSM, with a view towards systematically building in constraints on flavour-violating physics. Within this framework the space of parameters is reduced by expanding soft supersymmetry-breaking terms in powers of the Cabibbo angle, leading to a 24-, 30- or 42-parameter framework (which we call MSSM-24, MSSM-30, and MSSM-42 respectively), depending on the order kept in the expansion. We provide a Bayesian global fit to data of the MSSM-30 parameter set to show that this is manageable with current tools. We compare the MFV reductions to the 19-parameter pMSSM choice and show that the pMSSM is not contained as a subset. The MSSM-30 analysis favours a relatively lighter TeV-scale pseudoscalar Higgs boson and $\tan \beta \sim 10$ with multi-TeV sparticles.

KeYWords: Supersymmetry Phenomenology

ARXIV EPRINT: 1411.1663 


\section{Contents}

1 Introduction 1

2 The models 4

2.1 Parameter pruning 4

$\begin{array}{ll}2.2 & \text { Expansions in small mixing angles }\end{array}$

3 The MSSM-30 fit $\quad 8$

3.1 Fitting procedure 9

$\begin{array}{lll}3.2 & \text { Posterior distributions } & 11\end{array}$

4 Conclusions and outlook $\quad 12$

Don't panic.

(on the cover of The Hitchhiker's Guide to the Galaxy [1])

- Douglas Adams

\section{Introduction}

Supersymmetry, when linearly realised, requires the existence of superpartners to the known elementary particles, and robustly dictates their quantum numbers. Less robustly dictated are their masses and couplings once supersymmetry is spontaneously broken, as experiments demand it must be. A full description of these requires the more than 100 parameters of the supersymmetry-breaking sector of the R-parity conserving minimal supersymmetric Standard Model (MSSM).

The challenge of confronting such a vast parameter space with data drives the development of various kinds of well-motivated benchmark models. The earliest of these, the cMSSM/mSUGRA [2-4], specialises to a restricted parameter space motivated by what would be generated if supersymmetry were broken in a flavour-blind hidden sector (as suggested by the earliest gravity-mediation models). This simple model is one of the main benchmarks against which LHC results are compared, with the result that it is in real tension with the data.

But should this tension be regarded as evidence against supersymmetry, even if only in its linearly realised ${ }^{1}$ form? Answering this requires a more detailed exploration of the parameter space, yet a complete scan of the total parameters still remains beyond our current computational capabilities.

What is needed is a more strategic survey of the possibilities, of which several approaches have emerged. One approach — for example, Gauge-Mediated Supersymmetry

\footnotetext{
${ }^{1}$ See [5] for a well-motivated example where supersymmetry breaks at the electroweak scale but is nonlinearly realised in the Standard Model sector, and so doesn't require the existence of MSSM superpartners like squarks and sleptons.
} 
Breaking (GMSB) [6], or more sophisticated string-motivated gravity mediation mechanisms [7] — is to explore alternative mechanisms of supersymmetry breaking whose low-energy implications differ from those of the minimal gravity-mediated picture. Another focusses less on surveying the parameter space and more on the generic features of the underlying production and decay mechanisms, such as appear in 'simplified models' [8]. Comparison of such models to the data can quantify which of these mechanisms are favoured or disfavoured. A more specific 'simplified models' approach instead focuses on those interactions that take part in the naturalness issues that underlie the motivation for supersymmetry in the first place [9-11].

A third approach is to try to broadly survey the allowed parameter space, but to use prior knowledge about other constraints (like limits on flavour and CP violations) to cut down the range of parameters examined at the LHC. Of course this would be simple if it were just a matter of removing couplings that are excluded by other constraints. How the parameters are best pruned is more of a judgement call when the couplings of interest are not directly forbidden by other observations.

The phenomenological MSSM (pMSSM) [12] is one of the leading approaches along these lines which stakes out a 19-parameter subset of MSSM by removing all members of potentially dangerous families of couplings — such as all flavour-changing interactions beyond those already in the Standard Model (SM), for example. Besides providing a good motivation for dropping the discarded parameters, the remaining 19-parameter set is also broad enough to include many models and yet small enough to allow reasonably systematic comparisons with LHC data. On the other hand a drawback of the pMSSM is the relatively $a d-h o c$ way that the couplings are truncated in detail (in the precise sense described in more detail below).

Our goal in this paper is to proceed further along this line of reasoning, in particular to cast the removal of parameters in terms of approximate symmetries. This has the advantage of building in at the outset naturalness constraints since radiative corrections are guaranteed to respect the choices made for the assumed hierarchies amongst the model's parameters. In particular we use Minimal Flavour Violation (MFV) [13, 14, 14-49] as our main symmetry criterion to limit flavour-changing physics, wherein the flavour symmetries of the SM in the absence of Yukawa couplings are assumed to be broken only by other parameters that transform as do the SM Yukawa couplings themselves. In such a formulation all of the magic of the GIM mechanism [50] is automatically incorporated because flavour-changing interactions are typically suppressed by the same small mixing angles as are those of the SM fermions.

When imposed on the MSSM, the MFV hypothesis expresses flavour-violating supersymmetry-breaking interactions in new basis which emphasises their transformation properties under the approximate global flavour symmeties. This makes it possible to associate a power of the small symmetry-breaking size to all flavour-changing interactions in a way that is consistent with the known flavour-changes of the SM itself. Counting the suppression by this symmetry-breaking parameter provides a natural way to rank their size (and thereby gives a natural parameter-selection procedure, wherein one neglects all terms beyond a fixed order $[13-17,51])$. Of course, we are not the first to apply MFV methods 
to models beyond-the-SM (BSM) [14, 18-41] or to the MSSM [17, 42-49]. However, we believe our work represents the first use of MFV for systematic comparison of the MSSM to experiment.

Although very similar in spirit to the ideas behind the pMSSM, the MFV approach differs in detail and offers several advantages. An important advantage is the ability to strengthen (or weaken) the MFV parameter-selection prescription at will to exclude more (or fewer) interactions, simply by changing the order in symmetry breaking that is to be neglected. This is to be contrasted with setting all off-diagonal mass terms to zero by hand once and for all, as is done for the pMSSM. In this paper we consider three such choices: the strongest is a 24-parameter MSSM-24, which works at the lowest nontrivial order. At next order is a 30-parameter MSSM-30 and at the order beyond this lies a 42-parameter Adams' model, ${ }^{2}$ MSSM-42. Interestingly, none of these parameter sets contain the pMSSM, which is not defined by any fixed order in the MFV's small flavour-mixing parameter expansion.

All three of these are multi-parameter alternatives to the pMSSM. They contain all of the pMSSM's main virtue and more. They are broad enough to include a large variety of well-motivated supersymmetric model points, and yet are small enough to bring within reach a systematic comparison with experimental data. As a first illustration we perform such a comparison for MSSM-30, showing that even this 30-parameter system is not too large to be surveyed using reasonable resources. The systematic exploration of these models should provide a better way for drawing quantitative inferences regarding whether linearly realised supersymmetry is yet disfavoured by current data. As is also the case for the pMSSM, the broader set of parameters contains some atypical expectations compared to the simpler and more constrained sub-spaces usually considered, until recently, in supersymmetry searches. Although we focus only on R-parity invariant interactions, the method can be easily extended to include the R-parity violating MSSM [57-60].

From a bottom-up perspective, the drawback of using ad-hoc criteria for reducing the MSSM parameter space is the uncertainty of the theoretical prejudices that underlie the choices made. Selecting to work within a few-parameter framework comes with a cost - a potential loss of physics that may prove important. For example, moving from the cMSSM to the 20-parameter pMSSM, as done in refs. [52, 56], changed the favoured masses of the Higgs boson and the scalar top-quark to $119-128 \mathrm{GeV}$ and $2-3 \mathrm{TeV}$ respectively, at a time where such heavy masses were considered impossible within the traditional cMSSM. Another example: by setting the off-diagonal mass terms to be zero within the pMSSM frame, certain diagrams that contribute to flavour changing decays (such as in the decay $B_{s} \rightarrow \mu^{+} \mu^{-}$) are lost by construction.

The MFV framework provides a natural way to extend the number of parameters in a systematic fashion, order-by-order, from the traditional few-parameters towards the complete and phenomenological representations. We consider the work we present here as only a first step towards a more systematic approach to soft terms from a bottom-up perspective. Jumping from the handful of parameters of the cMSSM to phenomenological studies of the

\footnotetext{
${ }^{2}$ Which we name in honour of Douglas Adams, who predicted the importance of the number 42 for theoretical science [1].
} 
pMSSM took more than 25 years $[52-54,56]$ due in part to the computational challenge of considering more than 5 parameters. Thanks to increasing computing capacities this is becoming less of an issue.

The main disadvantage of a Bayesian analysis for models with many parameters is that prior-dependence can limit the predictive power. One possible approach in the short term is to seek observables that are prior-independent and to estimate, qualitatively, the extent at which current data is able to constrain the supersymmetry models $[65,67,68,70]$. In the longer term this is less of an issue as better, more constraining, data becomes available. In what follows we do not explore prior dependence in too much detail, beyond comparing some of our results with fits to the pMSSM, because our immediate goal is to define the general set-up for later use.

Our presentation is organised as follows. We first, in section 2 describe in more detail the choices made both in the pMSSM and in our three realisations of the MFV-MSSM. Section 3 then describes a global fit of the MSSM-30 model to the data, with the goal of illustrating the utility of the MFV approach. Finally section 4 briefly summarises our conclusions.

\section{The models}

In this section we provide a brief summary of the pMSSM and of the assumptions that go into the MFV-MSSMs that are compared later with observations. The starting point for both is the observation that a full comparison of LHC and other experiments data to the 100-plus parameters of the MSSM is not (yet) feasible, nor is it desirable (at the moment) given that many of these parameters describe processes that are strongly constrained by limits on flavour changing neutral currents (FCNCs) and on CP violation. Therefore we seek a methodology that allows a maximal probe of the MSSM parameter space with minimal imposition of ad-hoc relations or truncations amongst the free parameters.

\subsection{Parameter pruning}

We start with a broad-brush description of the pMSSM and MFV-MSSM, in particular showing how these are related to one another.

The pMSSM. The goal is to arrive at a criterion for excluding flavour-changing and CP-violating interactions. The pMSSM does so by making the following choices [12]:

- The absence of flavour-violating interactions (when renormalised at TeV scales);

- Degenerate masses and negligible Yukawa couplings for the first two generations of sfermions;

- No CP-violating interactions (beyond those of the SM CKM matrix);

- $R$-parity conservation;

- The lightest neutralino should be the lightest superpartner (LSP) and a thermal relic. 
This approach leads to a model for which 19 parameters capture superpartner and multiple-Higgs physics. The 19 parameters are: 10 sfermion masses $\left(m_{Q_{1}}, m_{Q_{3}}, m_{u_{1}}\right.$, $\left.m_{u_{3}}, m_{d_{1}}, m_{d_{3}}, m_{L_{1}}, m_{L_{3}}, m_{e_{1}}, m_{e_{3}}\right) ; 3$ gaugino masses $\left(M_{1}, M_{2}, M_{3}\right) ; 3$ trilinear scalar couplings $\left(A_{b}, A_{t}, A_{\tau}\right)$; and 3 Higgs/Higgsino parameters $\left(\mu, M_{A}, \tan \beta\right)$. We see that its definition includes choices that are well-motivated but ultimately ad-hoc. For instance, to avoid extra CP-violating sources the supersymmetry-breaking terms are set, by hand, to be real. This amounts to the assertion that no CP-violation effects play an important role in physical processes or interactions at colliders. Similarly, the first and second generation sfermion masses are set to be degenerate in order to avoid conflict with the non-observation of FCNCs, while the flavour changes of the SM are of course kept. It is necessarily tricky to distinguish BSM physics that explicitly violates flavour from the higher order corrections through which flavour-blind BSM physics learns about SM flavour violation.

An alternative approach is to systematically represent all flavour physics effects both SM and BSM - as a perturbation involving some natural flavour expansion parameter, such as would be the case in an MFV analysis. Although inspired by MFV considerations, the pMSSM flavour constraints are not derived using MFV symmetry considerations (though this claim is sometimes made).

Minimal flavour violation: the MFV-MSSM. The MFV hypothesis [13-16] formulates the small size of flavour-violating effects in terms of approximate symmetries. To this end the starting point is to identify the large group, $G$, of flavour symmetries that the SM enjoys when all Yukawa couplings vanish. The assumption is then that the only quantities that break these symmetries are spurion fields that are proportional to the SM Yukawa couplings themselves. That is, the action is $G$-invariant when expressed in terms of its regular fields and the spurion fields, with the spurion fields then being replaced by their vacuum expectation values, whose values are inferred from the SM Yukawa couplings. This has the virtue of automatically building in the GIM cancellations required by observations once loop effects are included.

As applied to the MSSM the upshot is that MFV boils down to the requirement that all the low-scale MSSM flavour couplings can be reconstructed entirely out of appropriate powers of the SM Yukawa coupling matrices, $Y_{U, D, E}$, ensuring that flavour violations are solely governed by the CKM matrix. Within the MFV framework, ${ }^{3}$ soft supersymmetry breaking terms are expanded in series of the $G$-invariant spurion factors [13-17, 51, 61, 72]:

$$
\begin{aligned}
\left(M_{Q}^{2}\right)_{i j} & =M_{Q}^{2}\left[\delta_{i j}+b_{1}\left(Y_{U}^{\dagger} Y_{U}\right)_{i j}+b_{2}\left(Y_{D}^{\dagger} Y_{D}\right)_{i j}+c_{1}\left\{\left(Y_{D}^{\dagger} Y_{D} Y_{U}^{\dagger} Y_{U}\right)_{i j}+H . c .\right\}+\ldots\right] \\
\left(M_{U}^{2}\right)_{i j} & =M_{U}^{2}\left[\delta_{i j}+b_{3}\left(Y_{U} Y_{U}^{\dagger}\right)_{i j}+\ldots\right] \\
\left(M_{D}^{2}\right)_{i j} & =M_{D}^{2}\left[\delta_{i j}+\left[Y_{D}\left(b_{6}+b_{7} Y_{U}^{\dagger} Y_{U}\right) Y_{D}^{\dagger}\right]_{i j}+\ldots\right] \\
\left(M_{L}^{2}\right)_{i j} & =M_{L}^{2}\left[\delta_{i j}+b_{13}\left(Y_{E}^{\dagger} Y_{E}\right)_{i j}+\ldots\right] \\
\left(M_{E}^{2}\right)_{i j} & =M_{E}^{2}\left[\delta_{i j}+b_{14}\left(Y_{E} Y_{E}^{\dagger}\right)_{i j}+\ldots\right]
\end{aligned}
$$

\footnotetext{
${ }^{3}$ There is also an alternative geometrical approach which is not considered here [55].
} 
and

$$
\begin{aligned}
& \left(A_{E}^{\prime}\right)_{i j}=a_{E}\left[\delta_{i j}+b_{15}\left(Y_{E}^{\dagger} Y_{E}\right)_{i j}+\ldots\right], \\
& \left(A_{U}^{\prime}\right)_{i j}=a_{U}\left[\delta_{i j}+b_{9}\left(Y_{U}^{\dagger} Y_{U}\right)_{i j}+b_{10}\left(Y_{D}^{\dagger} Y_{D}\right)_{i j}+\ldots\right], \\
& \left(A_{D}^{\prime}\right)_{i j}=a_{D}\left[\delta_{i j}+b_{11}\left(Y_{U}^{\dagger} Y_{U}\right)_{i j}+b_{12}\left(Y_{D}^{\dagger} Y_{D}\right)_{i j}+c_{6}\left(Y_{D}^{\dagger} Y_{D} Y_{U}^{\dagger} Y_{U}\right)_{i j}+\ldots\right] .
\end{aligned}
$$

Although the ellipses appear to denote an infinite series, this collapse to only a few terms due to the Cayley-Hamilton identities for $3 \times 3$ matrices. For instance, any generic matrix can be written in the form in eq. (2.1), but generically the required coefficients, $b_{i}$ and $c_{i}$, would span many orders of magnitude. The power of the MFV hypothesis lies in the assumption that the $b_{i}$ and $c_{i}$ are of order unity, with all small numbers suppressing flavour changes coming solely from those already in the Yukawa matrices. Trilinear scalar couplings similarly take the form $\left(A_{E, U, D}\right)_{i j}=\left(A_{E, U, D}^{\prime} Y_{E, U, D}\right)_{i j}$.

Now, a non-symmetry way to truncate the above parameters to a flavour-blind set is to impose $b_{i}=c_{i}=0$. This sets all off-diagonal elements of the matrices to zero and all diagonal elements are set to be equal to one another, leading to a 14-parameter flavourblind MSSM with no extra-SM sources of CP violation. Note these choices ensure the sfermion masses within each family are degenerate. Lifting the degeneracy to only the first two generations then gives the 19-parameter pMSSM. This shows how the pMSSM is related to the MFV MSSM, and why some of the assumptions in its construction do not rely on symmetries. By contrast, the number of MFV MSSM parameters in principle is the same as for the original MSSM, if we work to all orders in the small Yukawa couplings. However, within the MFV MSSM the number of parameters can be reduced in a systematic way by dropping terms smaller than a particular fixed order in small mixing angles (like the Cabibbo angle), as we now see.

\subsection{Expansions in small mixing angles}

A systematic approach for selecting the number of MSSM parameters have been prescribed in ref. [51]. The counting rule explores the hierarchical structure along the offdiagonals terms of the Yukawa matrices usually expressed in terms of the Cabibbo angle, $\lambda=\sin \theta_{C B} \simeq 0.23$. The idea starts from the observation that after the collapse of the infinite series in eq. (2.1) and eq. (2.2) into few terms by employing the Cayley-Hamilton identities, large pieces of the terms such as $\left(Y_{U}^{\dagger} Y_{U}\right)_{i j}^{2}$ and $\left(Y_{U}^{\dagger} Y_{U}\right)_{i j}$ are proportional to $V_{3 i}^{*} V_{3 j}$ where $V$ is the CKM matrix. The next relatively smaller terms are proportional to $V_{2 i}^{*} V_{2 j}$. So $V_{3 i}^{*} V_{3 j}$ and $V_{2 i}^{*} V_{2 j}$ can be used as basis vectors with coefficients of order one and $y_{c}^{2} \sim \lambda^{8}$ respectively instead of $\left(Y_{U}^{\dagger} Y_{U}\right)_{i j}^{2}$ and $\left(Y_{U}^{\dagger} Y_{U}\right)_{i j}$. Similarly, instead of $\left(Y_{D}^{\dagger} Y_{D}\right)_{i j}$ and $\left(Y_{D}^{\dagger} Y_{D}\right)_{i j}^{2}, \delta_{i 3}^{*} \delta_{j 3}$ and $\delta_{i 2}^{*} \delta_{j 2}$ can be used with order $y_{b}^{2}$ and $y_{s}^{2}$ coefficients respectively. Here $\delta_{i j}$ is the unit matrix in family space. This way, all possible multipliable structures lead to new complete basis vectors that form a closed algebra under multiplication:

$$
\begin{aligned}
& X_{1}=\delta_{3 i} \delta_{3 j}, \quad X_{2}=\delta_{2 i} \delta_{2 j}, \quad X_{3}=\delta_{3 i} \delta_{2 j}, \quad X_{4}=\delta_{2 i} \delta_{3 j}, \\
& X_{5}=\delta_{3 i} V_{3 j}, \quad X_{6}=\delta_{2 i} V_{2 j}, \quad X_{7}=\delta_{3 i} V_{2 j}, \quad X_{8}=\delta_{2 i} V_{3 j}, \\
& X_{9}=V_{3 i}^{*} \delta_{3 j}, \quad X_{10}=V_{2 i}^{*} \delta_{2 j}, \quad X_{11}=V_{3 i}^{*} \delta_{2 j}, \quad X_{12}=V_{2 i}^{*} \delta_{3 j}, \\
& X_{13}=V_{3 i}^{*} V_{3 j}, \quad X_{14}=V_{2 i}^{*} V_{2 j}, \quad X_{15}=V_{3 i}^{*} V_{2 j}, \quad X_{16}=V_{2 i}^{*} V_{3 j} \text {. }
\end{aligned}
$$


Note that the basis vectors are all of order one since each has at least one entry of order unity. With these, each of the MFV parameters can be assigned an order in $\lambda$. Once the accuracy of calculations is chosen in the form $\mathcal{O}\left(\lambda^{n}\right)$, then the prescription can be used to systematically discard terms within the supersymmetry-breaking parameters expansion expressed in the $X_{i}$ basis.

The MSSM-42 model. For instance, as done in ref. [51], dropping terms of order $\lambda^{6} \sim 10^{-4}$ and higher from the soft supersymmetry-breaking terms in eq. (2.1) and eq. (2.2), the MSSM parameters become:

$$
\begin{aligned}
e^{\phi_{1}} M_{1}, \quad e^{\phi_{2}} M_{2}, \quad M_{3}, \quad e^{\phi_{\mu}} \mu, \quad M_{A}, \quad \tan \beta \\
M_{Q}^{2}=\tilde{a}_{1}+x_{1} X_{13}+y_{1} X_{1}+y_{2} X_{5}+y_{2}^{*} X_{9}, \\
M_{U}^{2}=\tilde{a}_{2}+x_{2} X_{1}, \\
M_{D}^{2}=\tilde{a}_{3}+y_{3} X_{1}+w_{1} X_{3}+w_{1}^{*} X_{4}, \\
M_{L}^{2}=\tilde{a}_{6}+y_{6} X_{1}, \\
M_{E}^{2}=\tilde{a}_{7}+y_{7} X_{1}, \\
A_{E}=\tilde{a}_{8} X_{1}+w_{5} X_{2}, \\
A_{U}=\tilde{a}_{4} X_{5}+y_{4} X_{1}+w_{2} X_{6}, \\
A_{D}=\tilde{a}_{5} X_{1}+y_{5} X_{5}+w_{3} X_{2}+w_{4} X_{4} .
\end{aligned}
$$

Since the squark supersymmetry-breaking mass parameters are Hermitian then $\tilde{a}_{1-3,6,7}>$ $0, x_{1}, x_{2}, y_{1}, y_{3}, y_{6}, y_{7}$ must be real while the other coefficients can be complex. Hence the total number of supersymmetry-breaking parameters amounts to 42 , defining the MSSM- 42 .

The MSSM-30 model. Alternatively, keeping only those terms of order $\mathcal{O}\left(\lambda^{4}\right) \sim$ $\mathcal{O}\left(10^{-3}\right)$ means keeping only $x_{1-2}, y_{1}, y_{3}, y_{6}, y_{7} \in \mathbb{R}$; and $\tilde{a}_{4,5,8}, y_{4-5} \in \mathbb{C}$ terms from eq. (2.4). These make a 30-parameters MSSM-30:

$$
\begin{aligned}
e^{\phi_{1}} & M_{1}, \quad e^{\phi_{2}} M_{2}, \quad M_{3}, \quad \mu, \quad M_{A}, \quad \tan \beta, \quad e^{\phi_{\mu}} \\
M_{Q}^{2} & =\tilde{a}_{1}+x_{1} X_{13}+y_{1} X_{1} \\
M_{U}^{2} & =\tilde{a}_{2}+x_{2} X_{1} \\
M_{D}^{2} & =\tilde{a}_{3}+y_{3} X_{1} \\
M_{L}^{2} & =\tilde{a}_{6}+y_{6} X_{1} \\
M_{E}^{2} & =\tilde{a}_{7}+y_{7} X_{1} \\
A_{E} & =\tilde{a}_{8} X_{1} \\
A_{U} & =\tilde{a}_{4} X_{5}+y_{4} X_{1} \\
A_{D} & =\tilde{a}_{5} X_{1}+y_{5} X_{5}
\end{aligned}
$$

The MSSM-24 model. Going doing to $\mathcal{O}\left(\lambda^{3}\right) \sim \mathcal{O}\left(10^{-2}\right)$, only $x_{1-2} \in \mathbb{R}$; and $y_{5} \in \mathbb{C}$ remain from the non-diagonal mass and trilinear coupling expansion terms in eq. (2.5). 
These make a total of 24 soft-supersymmetry breaking parameters for MSSM-24:

$$
\begin{aligned}
e^{\phi_{1}} & M_{1}, \quad e^{\phi_{2}} M_{2}, \quad M_{3}, \quad \mu, \quad M_{A}, \quad \tan \beta, \quad e^{\phi_{\mu}} \\
M_{Q}^{2} & =\tilde{a}_{1}+x_{1} X_{13} \\
M_{U}^{2} & =\tilde{a}_{2}+x_{2} X_{1} \\
M_{D}^{2} & =\tilde{a}_{3} \\
M_{L}^{2} & =\tilde{a}_{6} \\
M_{E}^{2} & =\tilde{a}_{7} \\
A_{E} & =\tilde{a}_{8} X_{1} \\
A_{U} & =\tilde{a}_{4} X_{5} \\
A_{D} & =\tilde{a}_{5} X_{1}+y_{5} X_{5} .
\end{aligned}
$$

Note that the MFV MSSM parametrisation cannot be reduced to the 19 parameters of the pMSSM.

The MSSM-11 model. Ideally we would like to reduce the number of parameters even further, keeping the systematic approach we are following here. This we cannot do, but it is possible to define a minimal extension of the constrained MSSM - i.e. the cMSSM in a more $a d$ hoc way by setting in the above: $\phi_{1}=\phi_{2}=0, \tilde{a}_{1}=\tilde{a}_{2}=\tilde{a}_{3}=\tilde{a}_{6}=\tilde{a}_{7}=m_{0}$, $\operatorname{Re}\left(\tilde{a}_{8}\right)=\operatorname{Re}\left(\tilde{a}_{4}\right)=\operatorname{Re}\left(\tilde{a}_{5}\right)=A_{0}$, and $\operatorname{Im}\left(\tilde{a}_{8}\right)=\operatorname{Im}\left(\tilde{a}_{4}\right)=\operatorname{Im}\left(\tilde{a}_{5}\right)=0$. That is,

$$
\begin{aligned}
& M_{1}=M_{2}=M_{3}=m_{1 / 2}, \\
& \operatorname{Im}\left(m_{1 / 2}\right) \\
& \left\{\mu, M_{A}, e^{\phi_{\mu}}\right\} \rightarrow\left\{m_{H_{1}}=m_{H_{2}}=m_{0}, \operatorname{sign}(\mu)\right\}, \tan \beta, \\
& M_{Q}^{2}=m_{0}^{2}+x_{1} X_{13} \\
& M_{U}^{2}=m_{0}^{2}+x_{2} X_{1}, \quad M_{D}^{2}=M_{L}^{2}=M_{E}^{2}=m_{0}^{2}, \\
& A_{E}=A_{0} X_{1} \text {, } \\
& A_{U}=A_{0} X_{5} \text {, } \\
& A_{D}=A_{0} X_{1}+y_{5} X_{5}, \quad \operatorname{Im}\left(A_{0}, y_{5}\right) \text {. }
\end{aligned}
$$

This reduces the parameter space into an 11-parameters cMSSM or cMSSM-11. Given its simplicity it may be worth studying this model in detail even though it reintroduces some ad-hoc selection of parameters at the end.

Out of these sub-MSSMs derived via the MFV MSSM scheme, in this paper we concentrate on the MSSM-30 model and fit its parameters to experiments data as a first step into landscaping, and making further forecasts about, the MSSM parameter space.

\section{The MSSM-30 fit}

As mentioned earlier the MSSM-42 model cannot be reduced into the traditional pMSSM parameter space. The MSSM-24 is the closest to the pMSSM. However, looking at the parameter lists of the MSSM models mentioned in the previous section we select MSSM-30 for going beyond the pMSSM especially in the flavour sector. This is a first-step beyond the pMSSM within our series of MSSM projects [52, 56, 62-69] that is systematically built for absorbing experimental data from both energy- and intensity frontiers to high-energy 
physics explorations. The explorations of the MSSM CP-violating phases within various constructs can be found in the literature such as in refs. [51, 71-74]. The sub-MSSMs mainly fall into one of the the various constrained MSSMs, the pMSSM or flavour-blind MSSM with variable extra-SM CP phases. The MSSM-30 goes beyond these by construction, considering that the systematic inclusion of the flavour-violating terms is important, and by number of parameters. The procedure for the Bayesian fit of the MSSM-30 to data is described as follows.

\section{1 $\quad$ Fitting procedure}

We use Bayesian statistical methods for fitting the MSSM-30 to data. Bayes' theorem takes two input information for deriving essentially two inference about the model addressed. The process has to be within a well-defined context. The context, $\mathcal{H}$, for the MSSM-30 analysis is that the model represents R-parity preserving linearly realised supersymmetry and that the neutralino LSP make at least part of the cold dark matter (CDM) relic. One of the input is the assumption about the nature of the model parameters, $\underline{\theta}$. Here we assume a flat prior probability density, $p(\underline{\theta} \mid \mathcal{H})$, over the MSSM-30 parameters in eq. (2.5)

$$
\begin{gathered}
\underline{\theta} \equiv\left\{M_{1,2,3}, \mu, M_{A}, \tan \beta, \operatorname{Im}\left(M_{1,2}, \mu\right), \tilde{a}_{1,2, \ldots, 8},\right. \\
\left.\operatorname{Im}\left(\tilde{a}_{4,5,8}\right), x_{1,2}, y_{1,3,4,5,6,7}, \operatorname{Im}\left(y_{4,5}\right)\right\}
\end{gathered}
$$

where $M_{1}, M_{2}$ and $M_{3}$ are the gaugino mass parameters varied in the range -4 to $4 \mathrm{TeV}$ for both real and imaginary parts of $M_{1,2}$ and $100 \mathrm{GeV}$ to $4 \mathrm{TeV}$ for $M_{3}$. The massterm parameters $\tilde{a}_{1,2,3,6,7}>0$ are varied within the range $(100 \mathrm{GeV})^{2}$ to $(4 \mathrm{TeV})^{2}$ and $-(4 \mathrm{TeV})^{2}$ to $(4 \mathrm{TeV})^{2}$ for $x_{1,2}, y_{1,3,6,7}$. The trilinear coupling terms $\tilde{a}_{4,5,8}, \operatorname{Im}\left(\tilde{a}_{4,5,8}\right), y_{4,5}$, and $\operatorname{Im}\left(y_{4,5}\right)$ are varied within $-8 \mathrm{TeV}$ to $8 \mathrm{TeV}$. The Higgs-sector parameters are specified by the speudoscalar Higgs masses $M_{A}$, varied between $100 \mathrm{GeV}$ to $4 \mathrm{TeV}$ and the Higgs doublets mixing term $\mu, \operatorname{Im}(\mu)$ both varied within the range -4 to $4 \mathrm{TeV}$. The ratio of the vacuum expectation values $\tan \beta=\left\langle H_{2}\right\rangle /\left\langle H_{1}\right\rangle$ is allowed to be between 2 and $60 .{ }^{4}$ The SM parameters are fixed according to experimental results as: mass of the Z-boson, $m_{Z}=91.2 \mathrm{GeV}$, top quark mass, $m_{t}=165.4 \mathrm{GeV}$, bottom quark mass, $m_{b}=4.2 \mathrm{GeV}$, the electromagnetic coupling, $\alpha_{\mathrm{em}}^{-1}=127.9$, and the strong interaction coupling, $\alpha_{s}=0.119$.

The second input within Bayes' theorem is the data, $\underline{d}$. The data used for fitting the MSSM-30 are summarised in table 1. It is made of the experimental central values $\left(\mu_{i}\right)$ and errors $\left(\sigma_{i}\right)$ for the Higgs boson mass, the electroweak physics, B-physics, dipole moments of the leptons and the CDM relic density observables listed in the set $\underline{O}$ :

$$
\begin{gathered}
\underline{O} \equiv\left\{m_{h}, m_{W}, \Gamma_{Z}, \sin ^{2} \theta_{\mathrm{eff}}^{\mathrm{lep}}, R_{l}^{0}, R_{b, c}^{0}, A_{F B}^{b, c}, A^{l}=A^{e}, A^{b, c},\right. \\
\quad B R\left(B \rightarrow X_{s} \gamma\right), B R\left(B_{s} \rightarrow \mu^{+} \mu^{-}\right), \Delta M_{B_{s}}, R_{B R\left(B_{u} \rightarrow \tau \nu\right)}, \\
\left.\Omega_{\mathrm{CDM}} h^{2}, \operatorname{Br}\left(B_{d} \rightarrow \mu^{+} \mu^{-}\right), \Delta M_{B_{d}}, d_{e, \mu, \tau}\right\} .
\end{gathered}
$$

For this analysis, the constraint from anomalous magnetic dipole moment of the muon is not included in order to avoid possible tension with the EDM constraints since this have the potential of slowing down the exploration of the MSSM-30 parameter space.

\footnotetext{
${ }^{4} \mathrm{The} 4 \mathrm{TeV}$ range is taken having the $14 \mathrm{TeV}$ LHC capabilities in mind and also for consistency reasons (as well for the $\tan \beta$ range) to allow possible comparisons with our previous pMSSM work.
} 


\begin{tabular}{|ll|ll|}
\hline Observable & Constraint & Observable & Constraint \\
\hline$m_{W}[\mathrm{GeV}]$ & $80.399 \pm 0.023[75]$ & $A^{l}=A^{e}$ & $0.1513 \pm 0.0021[76]$ \\
$\Gamma_{Z}[\mathrm{GeV}]$ & $2.4952 \pm 0.0023[76]$ & $A^{b}$ & $0.923 \pm 0.020[76]$ \\
$\sin ^{2} \theta_{\mathrm{eff}}^{\text {lep }}$ & $0.2324 \pm 0.0012[76]$ & $A^{c}$ & $0.670 \pm 0.027[76]$ \\
$R_{l}^{0}$ & $20.767 \pm 0.025[76]$ & $B r\left(B_{s} \rightarrow \mu^{+} \mu^{-}\right)$ & $3.2_{-1.2}^{+1.5} \times 10^{-9}[77]$ \\
$R_{b}^{0}$ & $0.21629 \pm 0.00066[76]$ & $\Delta M_{B_{s}}$ & $17.77 \pm 0.12 \mathrm{ps}^{-1}[78]$ \\
$R_{c}^{0}$ & $0.1721 \pm 0.0030[76]$ & $R_{B r\left(B_{u} \rightarrow \tau \nu\right)}$ & $1.49 \pm 0.3091[79]$ \\
$A_{\mathrm{FB}}^{b}$ & $0.0992 \pm 0.0016[76]$ & $\Delta M_{B_{d}}$ & $0.507 \pm 0.005 \mathrm{ps}^{-1}[80]$ \\
$A_{\mathrm{FB}}^{c}$ & $0.0707 \pm 0.0035[76]$ & $\Omega_{\mathrm{CDM}} h^{2}$ & $0.11 \pm 0.02[81]$ \\
$m_{h}[\mathrm{GeV}]$ & $125.6 \pm 3.0[82,83]$ & $B r\left(B_{d} \rightarrow \mu^{+} \mu^{-}\right)$ & $<1.8 \times 10^{-8}[84]$ \\
$d_{\mu}$ & $<2.8 \times 10^{-19}[85]$ & $B r\left(B \rightarrow X_{s} \gamma\right)$ & $(3.52 \pm 0.25) \times 10^{-4}[86]$ \\
$d_{\tau}$ & $<1.1 \times 10^{-17}[87]$ & $d_{e}$ & $<1.6 \times 10^{-27}[88]$ \\
\hline
\end{tabular}

Table 1. Summary for the central values and errors for the electroweak physics, B-physics, dipole moments of the leptons and cold dark matter relic density constraints.

The compatibility of the MSSM-30 with the data is quantified at each point in parameter space by the likelihood, the probability of the data set given the parameter point, $p(\underline{d} \mid \underline{\theta}, \mathcal{H})$. Assuming the observables are independent,$^{5}$ the combined likelihood

$$
p(\underline{d} \mid \underline{\theta}, \mathcal{H})=L(x) \prod_{i} \frac{\exp \left[-\left(O_{i}-\mu_{i}\right)^{2} / 2 \sigma_{i}^{2}\right]}{\sqrt{2 \pi \sigma_{i}^{2}}}
$$

where the index $i$ runs over the list of observables $\underline{O}$, the variable $x$ represents the predicted value of neutralino CDM relic density at an MSSM-30 parameter point and

$$
L(x)=\left\{\begin{array}{ll}
1 /\left(y+\sqrt{\pi s^{2} / 2}\right) & \text { if } x<y \\
\exp \left[-(x-y)^{2} / 2 s^{2}\right] /\left(y+\sqrt{\pi s^{2} / 2}\right) & \text { if } x \geq y
\end{array} .\right.
$$

Here $y=0.11$ is the CDM relic density central value and $s=0.02$ the corresponding inflated (to allow for theoretical uncertainties) error.

The MSSM-30 parameters are passed to SPHENO [89, 90] and SUSY_FLAVOR [91] packages ${ }^{6}$ via the SLHA2 [94] interface, for computing the supersymmetry spectrum, mixing angles and couplings; and corresponding predictions: the branching ratios $B R\left(B_{s} \rightarrow\right.$ $\left.\mu^{+} \mu^{-}\right), B R(B \rightarrow s \gamma), R_{B R\left(B_{u} \rightarrow \tau \nu\right)}, B r\left(B_{d} \rightarrow \mu^{+} \mu^{-}\right), \Delta M_{B_{s}}, \Delta M_{B_{d}}$ and $d_{e, \mu, \tau}$. Using the SLHA1 [95] interface, the neutralino CDM relic density were computed using MICROMEGAs [96] while SUSYPOPE [97, 98] is used for computing precision observables that include the $W$-boson mass $m_{W}$, the effective leptonic mixing angle variable $\sin ^{2} \theta_{\mathrm{eff}}^{\text {lep }}$,

\footnotetext{
${ }^{5}$ We did not add the experimental correlations between some of the electroweak observables. This simplification is plausible since they are rather mildly constraining.

${ }^{6}$ Note that in this work no vacua analyses $[92,93]$ were carried out beyond those implemented in the spectrum calculators.
} 
the total $Z$-boson decay width, $\Gamma_{Z}$, and the other electroweak observables whose experimentally determined central values and associated errors are summarised in table 1 . The predictions from SUSY_FLAVOR were not used for fitting the MSSM-30 but could be used for comparing predictions from the two packages. The MultiNest $[99,100]$ package which implements the Nested Sampling algorithm [101] were used for fitting the MSSM-30 to data. The results of the Bayesian fit are the posterior probability density of the model parameters given the data, $p(\underline{\theta} \mid \underline{d}, \mathcal{H}$ ), and the support (or evidence), $\mathcal{Z}=p(\underline{d} \mid \mathcal{H})$, for the MSSM-30 from the data used. These come directly from Bayes' theorem

$$
p(\underline{\theta} \mid \underline{d}, \mathcal{H}) \times p(\underline{d} \mid \mathcal{H})=p(\underline{d} \mid \underline{\theta}, \mathcal{H}) \times p(\underline{\theta} \mid \mathcal{H}) .
$$

The posterior probability densities of the MSSM-30 parameters and representative sparticle masses are presented in the next subsection.

\subsection{Posterior distributions}

The quantities of interest to be investigated from the output of the MSSM-30 fit to data are the supersymmetry-breaking parameters and the sparticle masses. The former provide an indication of the preferred regions within the MSSM-30 hyperspace which are compatible with the experimental results while from the latter an insight could be obtained concerning the prospects for detecting sparticles at the LHC and/or future colliders.

The one-dimensional posterior probability distributions for the MSSM-30 parameters are shown in figure 1. The real and imaginary parts of the complex parameters are plotted on the same figure while the corresponding magnitudes and phases are shown in figure 2 . In addition we also present, in figure 2, the posterior distribution for the nature of the neutralino LSP's gaugino-Higgsino composition $\left(1-Z_{g}\right)$ where $Z_{g}=\left|N_{11}\right|^{2}+\left|N_{22}\right|^{2}$ with an LSP bino $\tilde{b}$, wino $\tilde{w}^{3}$ and Higgsinos $\tilde{H}_{1,2}$ combination

$$
\tilde{\chi}_{1}^{0}=N_{11} \tilde{b}+N_{12} \tilde{w}^{3}+N_{13} \tilde{H}_{1}^{0}+N_{14} \tilde{H}_{2}^{0}, \quad \sum_{i=1,2,3,4}\left(N_{1 i}\right)^{2}=1 .
$$

$N_{1 i}$ with $i=1,2,3,4$ are coefficient depending on the supersymmetry-breaking parameters [102]. The neutralino is dominantly Higgsino- or gaugino-like for $\left(1-Z_{g}\right)$ approximately equal to unity or zero respectively. The nature of the LSP composition in relevant for understanding the posterior distributions of the gauge-sector supersymmetry-breaking parameters.

From figure 2, it can be seen that the LSP and lightest chargino are quasi-degenerate, $m_{\chi_{1}^{ \pm}} \sim m_{\chi_{1}^{0}} \sim \mu$. Secondly, the posterior of distribution of $\left(1-Z_{g}\right)$ indicates that the LSP is mostly higgsino-like. Therefore there is an efficient neutralino-chargino co-annihilations taking place for satisfying the CDM relic density requirement. The posterior distributions for the gaugino mass parameters $M_{1}$ and $M_{2}$, and the electroweak symmetry breaking constraint control the nature of the neutralino gaugino higgsino admixtures. $M_{1}$ and $M_{2}$ remain approximately unconstrained because the scenario is similar to the cMSSM's focus point region, see e.g. [103], where the renormalisation group running of $m_{H_{2}}$ is decoupled from the gaugino and trilinear parameters. This is the case for the pMSSM distributions 
shown in dashed lines except for $M_{2}$ which looks quite different apparently due to the non-negligible interplay of the EDMs and other constrains on the imaginary parts $\operatorname{Im}\left(M_{1}\right)$ and $\operatorname{Im}\left(M_{2}\right)$ as shown in figure 1 or the corresponding phases $\left(\phi_{1,2}\right)$ shown in figure 2 . The gluino mass distribution is slightly preferred to be heavier relative to that in $[52,56]$ due to the intensity-frontier constraints.

Unlike the case for the gluino mass, the posterior distributions in figure 1 show that the intensity-frontier constraints, plus fixing $m_{h}=125 \pm 3 \mathrm{GeV}$ favour smaller values of $\tan \beta$ and lighter pseudoscalar Higgs boson mass $M_{A}$ relative to the fits in $[52,56]$. The $\tan \beta$ feature together with the tendencies for heavier gluinos and sparticles are compatible with the effect of the EDM constraints. The EDMs tend to be proportional to $\tan \beta[107]$ so the prevention of EDM over-production necessarily requires lower $\tan \beta$ values. The fit indicates a $95 \%$ credible interval (Bayesian confidence interval) of 4.5 to 26.9 with a mean value of $\langle\tan \beta\rangle=13.4 \pm 5.8$. The value of $M_{A}$ is within the range of 327.8 to $3803.3 \mathrm{GeV}$ at $95 \%$ credible interval with a mean-value $\left\langle M_{A}\right\rangle=1751.9 \pm 1024.5 \mathrm{GeV}$. The application of the ATLAS and CMS collaborations' search for MSSM Higgs bosons results [104, 105] on the MSSM-30 posterior would require a dedicated interpretation of their data within the new MSSM frame.

The remaining MSSM-30 parameters, which appear in the mass-squared terms, $\tilde{a}_{1,2,3,6,7}, x_{1,2}, y_{1,3,6,7}$ and those that appear in the trilinear couplings, $\tilde{a}_{4,5,8}, y_{4,5}$ cannot be compared due to their absence within the pMSSM. However, the mass-squared terms can be compared as shown in figure 2. It can be seen that the posterior sample from the flat-prior fit of the MSSM-30 to data favours supersymmetry-breaking parameters in regions deeper into the multi-TeV scale beyond the pMSSM results. This feature is expected for scenarios that alleviates the supersymmetry CP problems (see refs. [48, 106, 107] and references therein, for instance). The $\mathrm{CP}$-violating phases have to be either small or the sparticles be heavy into the multi-TeV regions. The phases were not restricted to be small for the MSSM-30 fit. The feature is also supported by the fact that radiative corrections to the lightest CP-even Higgs boson mass require heavy 3rd generation squarks for making up the constraint $m_{h}=125 \pm 3 \mathrm{GeV}$. The trilinear couplings, on another hand, are peaked around zero because values away tend to solutions with negative squark masses. The only exception here is for the leading parameter $\left(a_{4}, \operatorname{Im}\left(a_{4}\right)\right.$ in figure 1 and the corresponding magnitude $a_{4}$ and phase $\phi_{a_{4}}$ shown in figure 2) in the trilinear coupling term $A_{U}$ which is roughly fixed by the $m_{h}=125 \mathrm{GeV}$ constrain.

\section{Conclusions and outlook}

We have implemented the MFV hypothesis' reparametrisation of the R-parity conserving MSSM as a prescription for selecting supersymmetry-breaking parameters at various orders, $\mathcal{O}\left(\lambda^{n}\right), n=1,2,3, \ldots$, where $\lambda=\sin \theta_{C B}=0.23$ in a Cabibbo mixing angle $\left(\theta_{C B}\right)$ expansion of the flavour-violating mass and trilinear coupling terms. This leads to the construction of the phenomenological MSSM frames, namely MSSM-42 by keeping terms at order $\mathcal{O}\left(\lambda^{6}\right)$, MSSM-30 by keeping terms at order $\mathcal{O}\left(\lambda^{4}\right)$, and MSSM-24 by keeping terms at order $\mathcal{O}\left(\lambda^{3}\right)$ with 42,30 and 24 parameters respectively. The traditional pMSSM cannot be obtained 


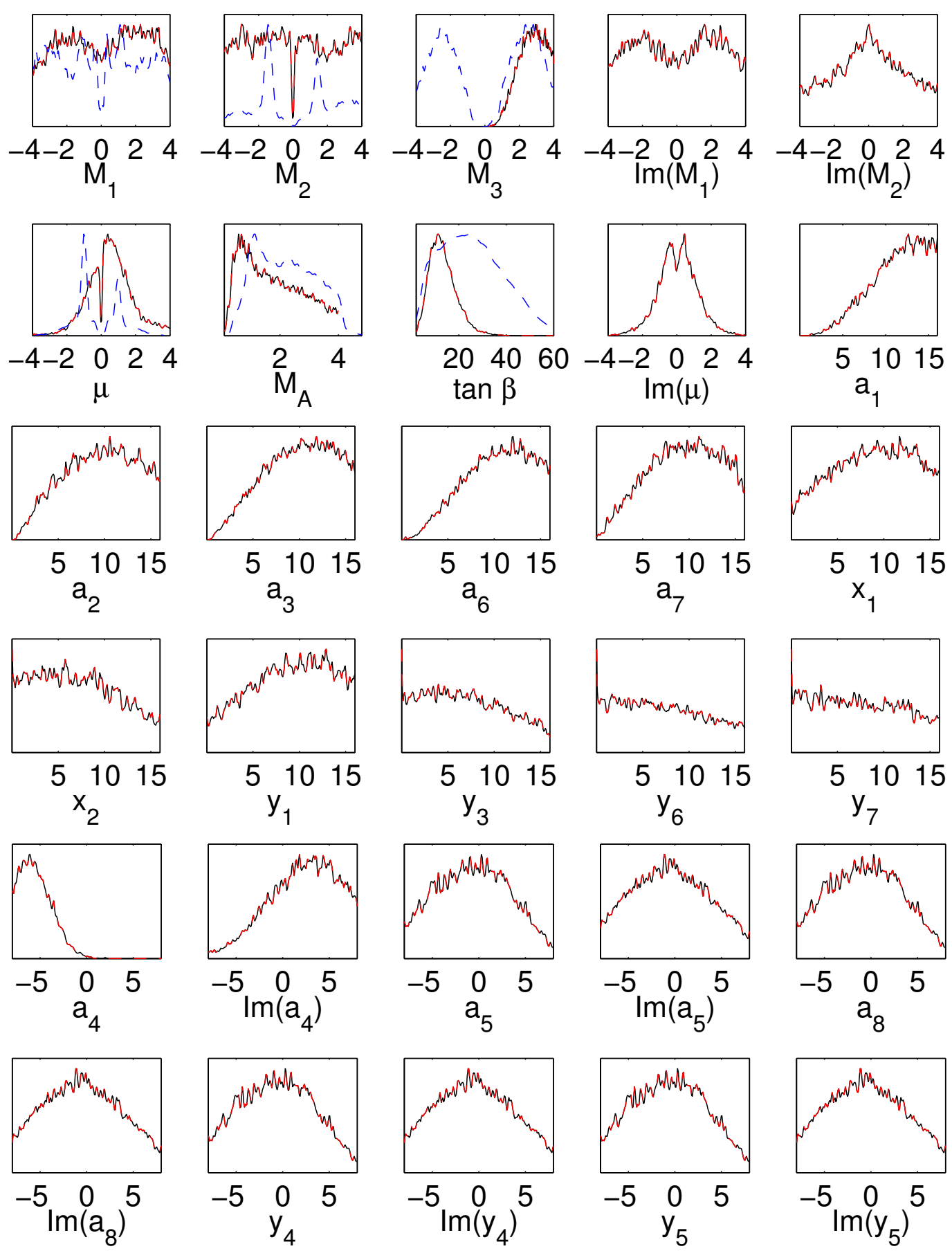

Figure 1. The plots show the posterior probability density functions, shown with solid curves, for the MSSM-30 parameters based on flat prior fit to the data in table 1. The dashed curves represent the corresponding posteriors for the 2008/9 pMSSM fits [52, 56] (when these are available) for comparison with the current MSSM-30 fit. The axes $M_{1,2,3}, \mu, M_{A}, \operatorname{Im}\left(M_{1,2}, \mu\right)$ are in TeV units. The parameters in the mass-squared terms, $\tilde{a}_{1,2,3,6,7}, x_{1,2}, y_{1,3,6,7}$ are in $\mathrm{TeV}^{2}$. The parameters entering the trilinear couplings $\tilde{a}_{4,5,8}, y_{4,5}$ are in $\mathrm{TeV}$ units. 

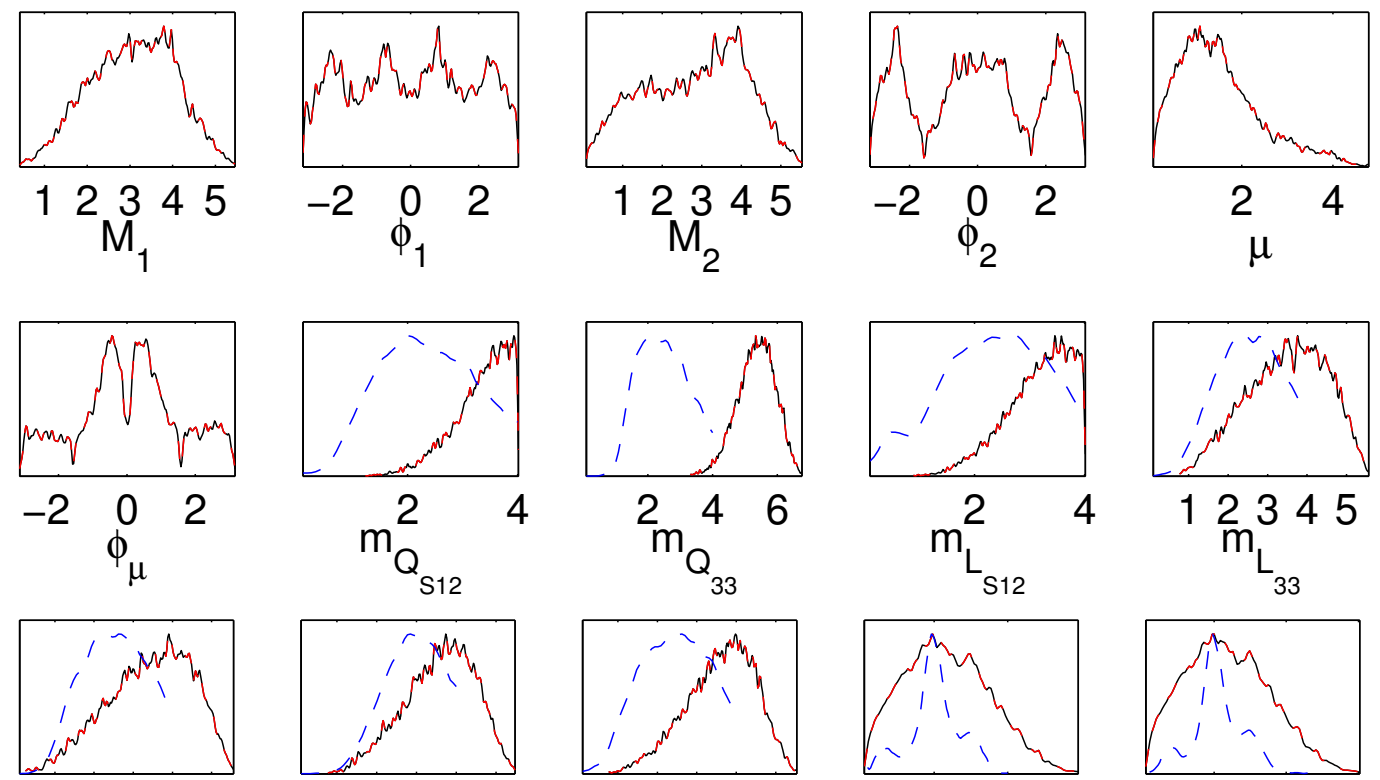

12345

$\mathrm{m}_{\mathrm{E}}$

12345
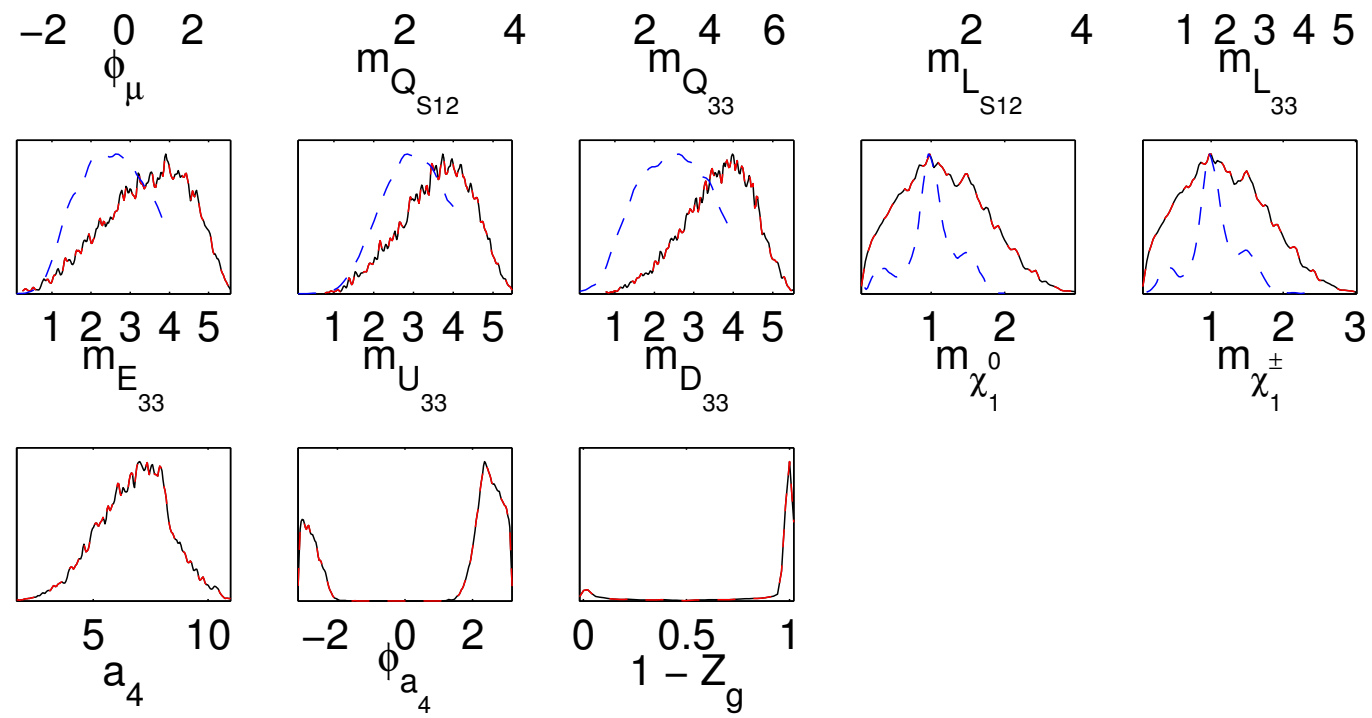

Figure 2. The solid line plots show the posterior distributions for the supersymmetry-breaking parameters derived from the base MSSM-30 parameters shown in figure 1. The dashed curves represent the corresponding posteriors for the 2008/9 pMSSM fits [52, 56] (when these are available) for comparison with the current MSSM-30 fit. The mass parameters are in $\mathrm{TeV}$ and the phases are in radians. The distribution of the neutralino composition $1-Z_{g}$ as described in the text is also shown.

via this systematic approach because by construction it has 1st-2nd generation squark mass degeneracies and off-diagonal elements in the mass terms set to zero by hand. The MSSM-42, MSSM-30, or MSSM-24 are suitable for fundamental physics studies involving the usually unavoidable energy- and intensity-frontier effects' interplay.

As a first step within our broader MSSM project, the MSSM-30 is chosen for going more significantly beyond the current R-parity conserving MSSM phenomenology constructs. The MSSM-30 parameters with $\mathcal{O}\left(\lambda^{4}\right) \sim \mathcal{O}\left(10^{-3}\right)$ coefficients in the MFV basis include the flavour conserving but CP-violating MSSM phases. We have performed a Bayesian global fit of the MSSM-30 to experiments data, following the standard techniques as in refs. $[52,56]$. The data consists of the Higgs boson mass, the electroweak physics, Bphysics, the electric dipole moments of the leptons and the CDM relic density observables. The posterior distributions of the 30 parameters are shown in figure 1 . The mass term posterior distributions shown in figure 2 indicate that the data used favours multi-TeV 1st/2nd generation and 3rd generation sparticles. 
The preference for smaller/lighter values of $\tan \beta$ and $M_{A}$ compared to the case for the $2008 / 9$ pMSSM fits $[52,56]$ is clear. Their posterior distributions are approximately prior-independent for the pMSSM fits $[52,56,108]$. This is also expected to be the case for the MSSM-30 since there is no feature or observable indicating otherwise, but the study of other priors is beyond the scope of the present paper. ${ }^{7}$ The MSSM-30 flat-prior fit indicates a $95 \%$ Bayesian confidence interval of 4.5 to 26.9 for $\tan \beta$ with a mean value of $\langle\tan \beta\rangle=13.4 \pm 5.8 . M_{A}$ is within the range of 327.8 to $3803.3 \mathrm{GeV}$ at $95 \%$ credible interval with a mean-value $\left\langle M_{A}\right\rangle=1751.9 \pm 1024.5 \mathrm{GeV}$. These numbers should be taken as indicative to complement the corresponding posterior distributions shown in figure 1. It would be interesting to assess the effect of the ATLAS and CMS's MSSM Higgs bosons search results $[104,105]$ on the MSSM-30 parameter space. This can be done by interpreting the experimental data within the MSSM-30 such as done in ref. [68] for interpreting the supersymmetry results within the pMSSM. It is also interesting to find the impact of direct and indirect dark matter detection data on the MSSM-30.

Extending our analysis to the more robust MSSM-42 should be achievable in the near future, including a comparison of different priors in order to extract prior-independent information. This is a concrete project to follow-up. This is especially relevant for future studies in search for supersymmetry with the LHC or some other future collider(s). The power of the Bayesian approach in determining prior-independent results should be applied within robust phenomenological frameworks such as the MSSM-30 and MSSM-42 for this purpose. Its relevance should improve with the increasing availability of data. Having preference for multi-TeV supersymmetric particles may also add to the different arguments supporting higher energy initiatives [109-111] such as a potential $100 \mathrm{TeV}$ machine [112].

\section{Acknowledgments}

We acknowledge useful discussions with Liliana Velasco-Sevilla on the SUSY_FLAVOUR SLHA-2 inputs and L. Aparicio, L. Silvestrini and K. Suruliz for discussions. S.S.A. would like to thank the School of Particles and Accelerators, IPM Tehran, for hospitality during the finishing phase of this paper. C.B. thanks the Abdus Salam International Centre for Theoretical Physics for its hospitality at various points during the completion of this work. The research leading to these results has received funding from the European Research Council under the European Union's Seventh Framework Programme (FP/20072013)/ERC Grant Agreement no. 279972 NPFlavour. C.B. is partially supported by grants from N.S.E.R.C. (Canada) and Perimeter Institute for Theoretical Physics. Research at Perimeter Institute is supported in part by the Government of Canada through Industry Canada, and by the Province of Ontario through the Ministry of Research and Information (MRI). This work was performed using the Darwin Supercomputer of the University of

\footnotetext{
${ }^{7}$ Notice that the comparison with the pMSSM, besides illustrating differences and similiarities of the outcomes of the two frameworks may also give us further information. Given the detailed analysis available for the pMSSM, for instance on the availability of different priors, we may also extract some information for the MSSM-30 scenario. Robust implications extracted from the pMSSM are not expected to be modified from the MSSM-30 analysis and therefore provide further information which is a net gain.
} 
Cambridge High Performance Computing Service (http://www.hpc.cam.ac.uk/), provided by Dell Inc. using Strategic Research Infrastructure Funding from the Higher Education Funding Council for England and funding from the Science and Technology Facilities Council.

Open Access. This article is distributed under the terms of the Creative Commons Attribution License (CC-BY 4.0), which permits any use, distribution and reproduction in any medium, provided the original author(s) and source are credited.

\section{References}

[1] D. Adams, The Hitchhiker's guide to the galaxy, Del Rey Publishing, U.K. (1995).

[2] S.P. Martin, A supersymmetry primer, Adv. Ser. Direct. High Energy Phys. 21 (2010) 1 [hep-ph/9709356] [INSPIRE].

[3] D.J.H. Chung et al., The soft supersymmetry breaking lagrangian: theory and applications, Phys. Rept. 407 (2005) 1 [hep-ph/0312378] [INSPIRE].

[4] P. Nath et al., The hunt for new physics at the Large Hadron Collider, Nucl. Phys. Proc. Suppl. 200-202 (2010) 185 [arXiv:1001.2693] [INSPIRE].

[5] C.P. Burgess, J. Matias and F. Quevedo, MSLED: a minimal supersymmetric large extra dimensions scenario, Nucl. Phys. B 706 (2005) 71 [hep-ph/0404135] [INSPIRE].

[6] G.F. Giudice and R. Rattazzi, Theories with gauge mediated supersymmetry breaking, Phys. Rept. 322 (1999) 419 [hep-ph/9801271] [INSPIRE].

[7] L.E. Ibanez and A.M. Uranga, String theory and particle physics: an introduction to string phenomenology, Cambridge University Press, Cambridge U.K. (2012).

[8] LhC New Physics Working Group collaboration, D. Alves et al., Simplified models for LHC new physics searches, J. Phys. G 39 (2012) 105005 [arXiv:1105.2838] [INSPIRE].

[9] R. Kitano and Y. Nomura, A solution to the supersymmetric fine-tuning problem within the MSSM, Phys. Lett. B 631 (2005) 58 [hep-ph/0509039] [INSPIRE].

[10] R. Kitano and Y. Nomura, Supersymmetry, naturalness and signatures at the LHC, Phys. Rev. D 73 (2006) 095004 [hep-ph/0602096] [INSPIRE].

[11] M. Papucci, J.T. Ruderman and A. Weiler, Natural SUSY endures, JHEP 09 (2012) 035 [arXiv:1110.6926] [INSPIRE].

[12] A. Djouadi, J.-L. Kneur and G. Moultaka, SuSpect: a Fortran code for the supersymmetric and Higgs particle spectrum in the MSSM, Comput. Phys. Commun. 176 (2007) 426 [hep-ph/0211331] [INSPIRE].

[13] R.S. Chivukula and H. Georgi, Composite technicolor standard model, Phys. Lett. B 188 (1987) 99 [INSPIRE].

[14] A.J. Buras, P. Gambino, M. Gorbahn, S. Jager and L. Silvestrini, Universal unitarity triangle and physics beyond the standard model, Phys. Lett. B 500 (2001) 161 [hep-ph/0007085] [INSPIRE].

[15] G. D'Ambrosio, G.F. Giudice, G. Isidori and A. Strumia, Minimal flavor violation: an effective field theory approach, Nucl. Phys. B 645 (2002) 155 [hep-ph/0207036] [INSPIRE]. 
[16] A.J. Buras, Minimal flavor violation, Acta Phys. Polon. B 34 (2003) 5615 [hep-ph/0310208] [INSPIRE].

[17] L.J. Hall and L. Randall, Weak scale effective supersymmetry, Phys. Rev. Lett. 65 (1990) 2939 [INSPIRE].

[18] M. Ciuchini, G. Degrassi, P. Gambino and G.F. Giudice, Next-to-leading QCD corrections to $B \rightarrow X_{s} \gamma$ in supersymmetry, Nucl. Phys. B 534 (1998) 3 [hep-ph/9806308] [INSPIRE].

[19] A. Ali and D. London, Profiles of the unitarity triangle and CP-violating phases in the standard model and supersymmetric theories, Eur. Phys. J. C 9 (1999) 687 [hep-ph/9903535] [INSPIRE].

[20] A.J. Buras and R. Fleischer, Bounds on the unitarity triangle, $\sin 2 \beta$ and $K \rightarrow$ neutrino anti-neutrino decays in models with minimal flavor violation, Phys. Rev. D 64 (2001) 115010 [hep-ph/0104238] [INSPIRE].

[21] C. Bobeth, T. Ewerth, F. Krüger and J. Urban, Enhancement of $B\left(\bar{B}_{d} \rightarrow \mu^{+} \mu^{-}\right) / B\left(\bar{B}_{s} \rightarrow \mu^{+} \mu^{-}\right)$in the MSSM with minimal flavor violation and large $\tan \beta$, Phys. Rev. D 66 (2002) 074021 [hep-ph/0204225] [INSPIRE].

[22] A.J. Buras, Relations between $\Delta M(s, d)$ and $B(s, d) \rightarrow \mu \bar{\mu}$ in models with minimal flavor violation, Phys. Lett. B 566 (2003) 115 [hep-ph/0303060] [INSPIRE].

[23] C. Bobeth et al., Upper bounds on rare $K$ and $B$ decays from minimal flavor violation, Nucl. Phys. B 726 (2005) 252 [hep-ph/0505110] [INSPIRE].

[24] V. Cirigliano, B. Grinstein, G. Isidori and M.B. Wise, Minimal flavor violation in the lepton sector, Nucl. Phys. B 728 (2005) 121 [hep-ph/0507001] [INSPIRE].

[25] K. Agashe, M. Papucci, G. Perez and D. Pirjol, Next to minimal flavor violation, hep-ph/0509117 [INSPIRE].

[26] UTfit collaboration, M. Bona et al., The UTfit collaboration report on the status of the unitarity triangle beyond the standard model. I. Model-independent analysis and minimal flavor violation, JHEP 03 (2006) 080 [hep-ph/0509219] [INSPIRE].

[27] B. Grinstein, V. Cirigliano, G. Isidori and M.B. Wise, Grand unification and the principle of minimal flavor violation, Nucl. Phys. B 763 (2007) 35 [hep-ph/0608123] [INSPIRE].

[28] Y. Grossman, Y. Nir, J. Thaler, T. Volansky and J. Zupan, Probing minimal flavor violation at the LHC, Phys. Rev. D 76 (2007) 096006 [arXiv:0706.1845] [INSPIRE].

[29] A.L. Fitzpatrick, G. Perez and L. Randall, Flavor anarchy in a Randall-Sundrum model with 5D minimal flavor violation and a low Kaluza-Klein scale, Phys. Rev. Lett. 100 (2008) 171604 [arXiv:0710.1869] [InSPIRE].

[30] G. Hiller and Y. Nir, Measuring flavor mixing with minimal flavor violation at the LHC, JHEP 03 (2008) 046 [arXiv:0802.0916] [InSPIRE].

[31] P. Paradisi, M. Ratz, R. Schieren and C. Simonetto, Running minimal flavor violation, Phys. Lett. B 668 (2008) 202 [arXiv:0805. 3989] [INSPIRE].

[32] T. Hurth, G. Isidori, J.F. Kamenik and F. Mescia, Constraints on new physics in MFV models: a model-independent analysis of $\Delta F=1$ processes, Nucl. Phys. B 808 (2009) 326 [arXiv: 0807.5039] [INSPIRE].

[33] A.L. Kagan, G. Perez, T. Volansky and J. Zupan, General minimal flavor violation, Phys. Rev. D 80 (2009) 076002 [arXiv:0903.1794] [INSPIRE]. 
[34] C.P. Burgess, M. Trott and S. Zuberi, Light octet scalars, a heavy Higgs and minimal flavour violation, JHEP 09 (2009) 082 [arXiv:0907.2696] [INSPIRE].

[35] J.M. Arnold, M. Pospelov, M. Trott and M.B. Wise, Scalar representations and minimal flavor violation, JHEP 01 (2010) 073 [arXiv:0911.2225] [INSPIRE].

[36] J.M. Arnold, B. Fornal and M. Trott, Prospects and constraints for vector-like MFV matter at LHC, JHEP 08 (2010) 059 [arXiv: 1005.2185] [INSPIRE].

[37] B. Batell and M. Pospelov, $B_{s}$ mixing and electric dipole moments in MFV, Phys. Rev. D 82 (2010) 054033 [arXiv:1006.2127] [INSPIRE].

[38] B. Batell, J. Pradler and M. Spannowsky, Dark matter from minimal flavor violation, JHEP 08 (2011) 038 [arXiv: 1105.1781] [INSPIRE].

[39] M. Redi, Composite MFV and beyond, Eur. Phys. J. C 72 (2012) 2030 [arXiv:1203.4220] [INSPIRE].

[40] W. Altmannshofer, S. Gori and G.D. Kribs, A minimal flavor violating 2HDM at the LHC, Phys. Rev. D 86 (2012) 115009 [arXiv:1210.2465] [INSPIRE].

[41] G. Krnjaic and D. Stolarski, Gauging the way to MFV, JHEP 04 (2013) 064 [arXiv:1212.4860] [INSPIRE].

[42] A. Ali and E. Lunghi, Extended minimal flavor violating MSSM and implications for B physics, Eur. Phys. J. C 21 (2001) 683 [hep-ph/0105200] [INSPIRE].

[43] S. Heinemeyer, W. Hollik, F. Merz and S. Penaranda, Electroweak precision observables in the MSSM with nonminimal flavor violation, Eur. Phys. J. C 37 (2004) 481 [hep-ph/0403228] [INSPIRE].

[44] G. Degrassi, P. Gambino and P. Slavich, QCD corrections to radiative B decays in the MSSM with minimal flavor violation, Phys. Lett. B 635 (2006) 335 [hep-ph/0601135] [INSPIRE].

[45] W. Altmannshofer, A.J. Buras and D. Guadagnoli, The MFV limit of the MSSM for low $\tan \beta$ : meson mixings revisited, JHEP 11 (2007) 065 [hep-ph/0703200] [INSPIRE].

[46] G. Degrassi, P. Gambino and P. Slavich, SusyBSG: a Fortran code for BR $\left[B \rightarrow X_{s} \gamma\right]$ in the MSSM with minimal flavor violation, Comput. Phys. Commun. 179 (2008) 759 [arXiv: 0712.3265] [INSPIRE].

[47] M. Carena, A. Menon and C.E.M. Wagner, Minimal flavor violation and the scale of supersymmetry breaking, Phys. Rev. D 79 (2009) 075025 [arXiv:0812.3594] [InSPIRE].

[48] P. Paradisi and D.M. Straub, The SUSY CP problem and the MFV principle, Phys. Lett. B 684 (2010) 147 [arXiv:0906.4551] [INSPIRE].

[49] J. Berger, C. Csáki, Y. Grossman and B. Heidenreich, Mesino oscillation in MFV SUSY, Eur. Phys. J. C 73 (2013) 2408 [arXiv:1209.4645] [INSPIRE].

[50] S.L. Glashow, J. Iliopoulos and L. Maiani, Weak interactions with lepton-hadron symmetry, Phys. Rev. D 2 (1970) 1285 [inSPIRE].

[51] G. Colangelo, E. Nikolidakis and C. Smith, Supersymmetric models with minimal flavour violation and their running, Eur. Phys. J. C 59 (2009) 75 [arXiv:0807.0801] [INSPIRE].

[52] S.S. AbdusSalam, The full 24-parameter MSSM exploration, AIP Conf. Proc. 1078 (2009) 297 [arXiv:0809. 0284] [INSPIRE]. 
[53] C.F. Berger, J.S. Gainer, J.L. Hewett and T.G. Rizzo, Supersymmetry without prejudice, JHEP 02 (2009) 023 [arXiv:0812.0980] [InSPIRE].

[54] M.W. Cahill-Rowley, J.L. Hewett, A. Ismail and T.G. Rizzo, More energy, more searches, but the phenomenological MSSM lives on, Phys. Rev. D 88 (2013) 035002 [arXiv:1211.1981] [INSPIRE].

[55] J. Ellis, R.N. Hodgkinson, J.S. Lee and A. Pilaftsis, Flavour geometry and effective Yukawa couplings in the MSSM, JHEP 02 (2010) 016 [arXiv:0911.3611] [INSPIRE].

[56] S.S. AbdusSalam, B.C. Allanach, F. Quevedo, F. Feroz and M. Hobson, Fitting the phenomenological MSSM, Phys. Rev. D 81 (2010) 095012 [arXiv:0904.2548] [INSPIRE].

[57] E. Nikolidakis and C. Smith, Minimal flavor violation, seesaw and R-parity, Phys. Rev. D 77 (2008) 015021 [arXiv:0710.3129] [INSPIRE].

[58] C. Csáki, Y. Grossman and B. Heidenreich, MFV SUSY: a natural theory for R-parity violation, Phys. Rev. D 85 (2012) 095009 [arXiv:1111.1239] [INSPIRE].

[59] J. Berger, M. Perelstein, M. Saelim and P. Tanedo, The same-sign dilepton signature of RPV/MFV SUSY, JHEP 04 (2013) 077 [arXiv: 1302.2146] [INSPIRE].

[60] G. Arcadi, L. Di Luzio and M. Nardecchia, Minimal flavour violation and neutrino masses without R-parity, JHEP 05 (2012) 048 [arXiv:1111.3941] [INSPIRE].

[61] C. Smith, Minimal flavor violation in supersymmetric theories, Acta Phys. Polon. Supp. 3 (2010) 53 [arXiv:0909.4444] [inSPIRE].

[62] F. Feroz et al., Bayesian selection of $\operatorname{sign}(\mu)$ within $m S U G R A$ in global fits including WMAP5 results, JHEP 10 (2008) 064 [arXiv:0807.4512] [INSPIRE].

[63] S.S. AbdusSalam, B.C. Allanach, M.J. Dolan, F. Feroz and M.P. Hobson, Selecting a model of supersymmetry breaking mediation, Phys. Rev. D 80 (2009) 035017 [arXiv:0906.0957] [INSPIRE].

[64] S.S. AbdusSalam and F. Quevedo, Cold dark matter hypotheses in the MSSM, Phys. Lett. B 700 (2011) 343 [arXiv: 1009.4308] [INSPIRE].

[65] S.S. AbdusSalam, Can the LHC rule out the MSSM?, Phys. Lett. B 705 (2011) 331 [arXiv:1106.2317] [INSPIRE].

[66] S.S. AbdusSalam et al., Benchmark models, planes, lines and points for future SUSY searches at the LHC, Eur. Phys. J. C 71 (2011) 1835 [arXiv:1109.3859] [INSPIRE].

[67] S.S. AbdusSalam and D. Choudhury, Higgs boson discovery versus sparticles prediction: Impact on the pMSSM's posterior samples from a Bayesian global fit, UJPA 2 (2014) 155 [arXiv: 1210.3331] [INSPIRE].

[68] S.S. AbdusSalam, LHC-7 supersymmetry search interpretation within the phenomenological MSSM, Phys. Rev. D 87 (2013) 115012 [arXiv:1211.0999] [INSPIRE].

[69] S.S. AbdusSalam, Stop-mass prediction in naturalness scenarios within MSSM-25, Int. J. Mod. Phys. A 29 (2014) 1450160 [arXiv:1312.7830] [InSPIRE].

[70] S. Sekmen et al., Interpreting LHC SUSY searches in the phenomenological MSSM, JHEP 02 (2012) 075 [arXiv:1109.5119] [INSPIRE].

[71] A. Bartl et al., General flavor blind MSSM and CP-violation, Phys. Rev. D 64 (2001) 076009 [hep-ph/0103324] [INSPIRE]. 
[72] J.R. Ellis, J.S. Lee and A. Pilaftsis, B-meson observables in the maximally CP-Violating MSSM with minimal flavour violation, Phys. Rev. D 76 (2007) 115011 [arXiv:0708.2079] [INSPIRE].

[73] L. Mercolli and C. Smith, EDM constraints on flavored CP-violating phases, Nucl. Phys. B 817 (2009) 1 [arXiv:0902.1949] [InSPIRE].

[74] J. Berger et al., The CP-violating pMSSM at the intensity frontier, arXiv:1309.7653 [INSPIRE].

[75] M. Verzocchi, Electroweak physics, talk gven at $34^{\text {th }}$ International Conference on High Energy Physics (ICHEP2008), July 30-August 5, Philadelphia, U.S.A. (2008).

[76] ALEPH, DELPHI, L3, OPAL, SLD, LEP Electroweak Working Group, SLD Electroweak Group, SLD Heavy Flavour Group collaboration, S. Schael et al., Precision electroweak measurements on the $Z$ resonance, Phys. Rept. 427 (2006) 257 [hep-ex/0509008] [INSPIRE].

[77] LHCb collaboration, First evidence for the decay $B_{s}^{0} \rightarrow \mu^{+} \mu^{-}$, Phys. Rev. Lett. 110 (2013) 021801 [arXiv:1211.2674] [INSPIRE].

[78] CDF collaboration, A. Abulencia et al., Observation of $B_{s}^{0}-\bar{B}_{s}^{0}$ oscillations, Phys. Rev. Lett. 97 (2006) 242003 [hep-ex/0609040] [INSPIRE].

[79] BABAR collaboration, B. Aubert et al., Search for the rare leptonic decay $B^{-} \rightarrow \tau^{-} \bar{\nu}_{\tau}$, Phys. Rev. Lett. 95 (2005) 041804 [hep-ex/0407038] [InSPIRE].

[80] Heavy Flavor Averaging Group collaboration, E. Barberio et al., Averages of b-hadron and c-hadron properties at the end of 200\%, arXiv:0808.1297 [INSPIRE].

[81] WMAP collaboration, E. Komatsu et al., Five-year Wilkinson Microwave Anisotropy Probe (WMAP) observations: cosmological interpretation, Astrophys. J. Suppl. 180 (2009) 330 [arXiv: 0803.0547] [INSPIRE].

[82] ATLAS collaboration, Combined measurements of the mass and signal strength of the Higgs-like boson with the ATLAS detector using up to $25 \mathrm{fb}^{-1}$ of proton-proton collision data, ATLAS-CONF-2013-014 (2013).

[83] CMS collaboration, Combination of standard model Higgs boson searches and measurements of the properties of the new boson with a mass near $125 \mathrm{GeV}$, CMS-PAS-HIG-13-005 (2013).

[84] LHCb collaboration, Search for the rare decays $B_{s}^{0} \rightarrow \mu^{+} \mu^{-}$and $B^{0} \rightarrow \mu^{+} \mu^{-}$, Phys. Lett. B 699 (2011) 330 [arXiv:1103.2465] [INSPIRE].

[85] Muon G-2 collaboration, R. McNabb, An improved limit on the electric dipole moment of the muon, hep-ex/0407008 [INSPIRE].

[86] Heavy Flavor Averaging Group collaboration, E. Barberio et al., Averages of b-hadron properties at the end of 2006, arXiv:0704.3575 [INSPIRE].

[87] Particle Data Group collaboration, K. Nakamura et al., Review of particle physics, J. Phys. G 37 (2010) 075021 [INSPIRE].

[88] B.C. Regan, E.D. Commins, C.J. Schmidt and D. DeMille, New limit on the electron electric dipole moment, Phys. Rev. Lett. 88 (2002) 071805 [INSPIRE].

[89] W. Porod, SPheno, a program for calculating supersymmetric spectra, SUSY particle decays and SUSY particle production at $e^{+} e^{-}$colliders, Comput. Phys. Commun. 153 (2003) 275 [hep-ph/0301101] [INSPIRE]. 
[90] W. Porod and F. Staub, SPheno 3.1: extensions including flavour, CP-phases and models beyond the MSSM, Comput. Phys. Commun. 183 (2012) 2458 [arXiv:1104.1573] [INSPIRE].

[91] A. Crivellin et al., SUSY_FLAVOR v2: a computational tool for FCNC and CP-violating processes in the MSSM, Comput. Phys. Commun. 184 (2013) 1004 [arXiv:1203.5023] [INSPIRE].

[92] J.E. Camargo-Molina, B. O'Leary, W. Porod and F. Staub, Vevacious: a tool for finding the global minima of one-loop effective potentials with many scalars,

Eur. Phys. J. C 73 (2013) 2588 [arXiv:1307.1477] [INSPIRE].

[93] J.E. Camargo-Molina, B. Garbrecht, B. O'Leary, W. Porod and F. Staub, Constraining the natural MSSM through tunneling to color-breaking vacua at zero and non-zero temperature, Phys. Lett. B 737 (2014) 156 [arXiv:1405.7376] [INSPIRE].

[94] B.C. Allanach et al., SUSY Les Houches accord 2, Comput. Phys. Commun. 180 (2009) 8 [arXiv:0801.0045] [INSPIRE].

[95] P.Z. Skands et al., SUSY Les Houches accord: Interfacing SUSY spectrum calculators, decay packages and event generators, JHEP 07 (2004) 036 [hep-ph/0311123] [INSPIRE].

[96] G. Bélanger, F. Boudjema, A. Pukhov and A. Semenov, Dark matter direct detection rate in a generic model with MicrOMEGAs 2.2, Comput. Phys. Commun. 180 (2009) 747 [arXiv:0803.2360] [INSPIRE].

[97] S. Heinemeyer, W. Hollik, D. Stöckinger, A.M. Weber and G. Weiglein, Precise prediction for $M(W)$ in the MSSM, JHEP 08 (2006) 052 [hep-ph/0604147] [INSPIRE].

[98] S. Heinemeyer, W. Hollik, A.M. Weber and G. Weiglein, Z pole observables in the MSSM, JHEP 04 (2008) 039 [arXiv:0710.2972] [INSPIRE].

[99] F. Feroz and M.P. Hobson, Multimodal nested sampling: an efficient and robust alternative to $M C M C$ methods for astronomical data analysis,

Mon. Not. Roy. Astron. Soc. 384 (2008) 449 [arXiv:0704.3704] [InSPIRE].

[100] F. Feroz, M.P. Hobson and M. Bridges, MultiNest: an efficient and robust Bayesian inference tool for cosmology and particle physics,

Mon. Not. Roy. Astron. Soc. 398 (2009) 1601 [arXiv:0809.3437] [InSPIRE].

[101] J. Skilling, Nested sampling, AIP Proc. 735 (2004) 395.

[102] M.M. El Kheishen, A.A. Aboshousha and A.A. Shafik, Analytic formulas for the neutralino masses and the neutralino mixing matrix, Phys. Rev. D 45 (1992) 4345 [INSPIRE].

[103] J.L. Feng, K.T. Matchev and T. Moroi, Focus points and naturalness in supersymmetry, Phys. Rev. D 61 (2000) 075005 [hep-ph/9909334] [InSPIRE].

[104] ATLAS collaboration, Search for neutral Higgs bosons of the minimal supersymmetric standard model in pp collisions at $\sqrt{s}=8 \mathrm{TeV}$ with the ATLAS detector, JHEP 11 (2014) 056 [arXiv:1409.6064] [INSPIRE].

[105] CMS collaboration, Search for neutral MSSM Higgs bosons decaying to a pair of $\tau$ leptons in pp collisions, JHEP 10 (2014) 160 [arXiv:1408.3316] [INSPIRE].

[106] A.G. Cohen, D.B. Kaplan and A.E. Nelson, The more minimal supersymmetric standard model, Phys. Lett. B 388 (1996) 588 [hep-ph/9607394] [INSPIRE]. 
[107] M. Pospelov and A. Ritz, Electric dipole moments as probes of new physics, Annals Phys. 318 (2005) 119 [hep-ph/0504231] [INSPIRE].

[108] S. AbdusSalam, B. Allanach, F. Quevedo, F. Feroz and M. Hobson, The posterior sample points' SLHA files from the Bayesian fits of the phenomenological MSSM, Harvard Dataverse Network (2013).

[109] The International Linear Collider technical design report, volumes 1-5, ILC-REPORT-2013-040 (2013).

[110] Future Circular Collider (FCC) study, http://cern.ch/fcc.

[111] M. Aicheler et al., A multi-TeV linear collider based on CLIC technology - CLIC conceptual design report, CERN-2012-007 (2012).

[112] A. Fowlie and M. Raidal, Prospects for constrained supersymmetry at $\sqrt{s}=33 \mathrm{TeV}$ and $\sqrt{s}=100$ TeV proton-proton super-colliders, Eur. Phys. J. C 74 (2014) 2948 [arXiv:1402.5419] [INSPIRE]. 